02,09

\title{
Система терагерцевой спектроскопии газовых смесей на основе твердотельных сверхпроводниковых источника и приемника терагерцевого диапазона
}

\author{
(C) Н.В. Кинев ${ }^{1}$, К.И. Рудаков ${ }^{1,2}$, Л.В. Филиппенко ${ }^{1}$, В.П. Кошелец ${ }^{1}$ \\ ${ }^{1}$ Институт радиотехники и электроники им. В.А. Котельникова РАН, \\ Москва, Россия \\ ${ }^{2}$ Астрономический институт Университета Гронингена, \\ Гронинген, Нидерланды \\ E-mail: nickolay@hitech.cplire.ru \\ Поступила в Редакцию 9 апреля 2021 г. \\ В окончательной редакции 9 апреля 2021 г. \\ Принята к публикации 19 апреля 2021 г.
}

\begin{abstract}
Продемонстрировано применение джозефсоновского генератора терагерцового диапазона на основе распределенного туннельного перехода „сверхпроводник-изолятор-сверхпроводник“, согласованного с передающей антенной и излучающего сигнал в открытое пространство, для спектроскопии газов. Генератор использован в качестве активного источника, сигнал которого поглощается образцом газовой смеси в ячейке длиной $60 \mathrm{~cm}$ и затем регистрируется спектрометром на основе приемника „сверхпроводникизолятор-сверхпроводник“ со спектральным разрешением лучше $100 \mathrm{kHz}$. В ходе эксперимента зарегистрированы линии поглощения аммиака и воды в терагерцевом диапазоне, а также продемонстрирована зависимость спектральных характеристик линий поглощения от давления газовой смеси в широких пределах (от 0.005 до 10 mbar).
\end{abstract}

Ключевые слова: источники и приемники терагерцевого диапазона, эффект Джозефсона, спектроскопия поглощения, газовый анализ.

DOI: 10.21883/FTT.2021.09.51239.03H

\section{1. Введение}

Спектроскопия в терагерцевом (ТГц) диапазоне на сегодняшний день активно применяется в широком спектре задач, от исследования физики конденсированного состояния до построения биомедицинских изображений. Существуют книги и обзоры, всецело посвященные принципам и применениям данного направления [1-3]. Так, ТГц-спектроскопия используется в физике и материаловедении для изучения динамики переноса заряда в различных полупроводниковых структурах - гетеропереходах, квантовых точках, нанокристаллах [1,2], в сверхпроводниках и сильно коррелированных электронных системах [1]. Методами ТГц-спектроскопии исследуются процессы электрической проводимости и фотопроводимости в различных материалах [3]. Применения в аналитической химии и медицине включают в себя спектроскопию биологических тканей и образцов, фармацевтические исследования [3]. ТГц-излучение используется в системах безопасности для обнаружения взрывчатых и запрещенных веществ [4] и для построения изображений в ТГц-диапазоне [2,3]. Поскольку ТГц-излучение является неразрушающим, его активно используют в биологических системах для исследования колебательных мод, которые распространяются через большие части биомолекулярных структур (белков, РНК, ДНК) с масштабами длины до десятков ангстрем [5]. Исследуемые явления важны для функционирования биоматериалов, которые претерпевают трансформации во вторичных и третичных структурах.

Столь широкий круг областей применения ТГцспектроскопии стимулирует создание новых методов и приборов. Некоторое время назад нашей группой была предложена и реализована концепция генератора в открытое пространство на основе распределенного джозефсоновского перехода (РДП) вида „сверхпроводник-изолятор-сверхпроводник“ (СИС), согласованного на одной микросхеме с передающей антенной и гармоническим смесителем (ГС) для фазовой стабилизации выходного сигнала [6-10]. Рабочая частота источника $f$ управляется в достаточно широких пределах постоянным напряжением смещения на переходе $V$, связанным с частотой посредством джозефсоновской константы $483.6 \mathrm{GHz} / \mathrm{mV}$ :

$$
f=(2 e / h) \cdot V,
$$

где $h$ - постоянная Планка, $e-$ заряд электрона. Мощность излучения такого генератора составляет от сотен нановатт до единиц микроватт [11] в спектральной полосе шириной от сотен килогерц до десятка мегагерц в автономном режиме генерации. Для сужения спектральной линии излучения традиционно используется система фазовой автоподстройки частоты (ФАПЧ), стабилизирующая до $97 \%$ мощности в узком пике шириной порядка $40 \mathrm{kHz}$. Частота генерации составляет от 250 


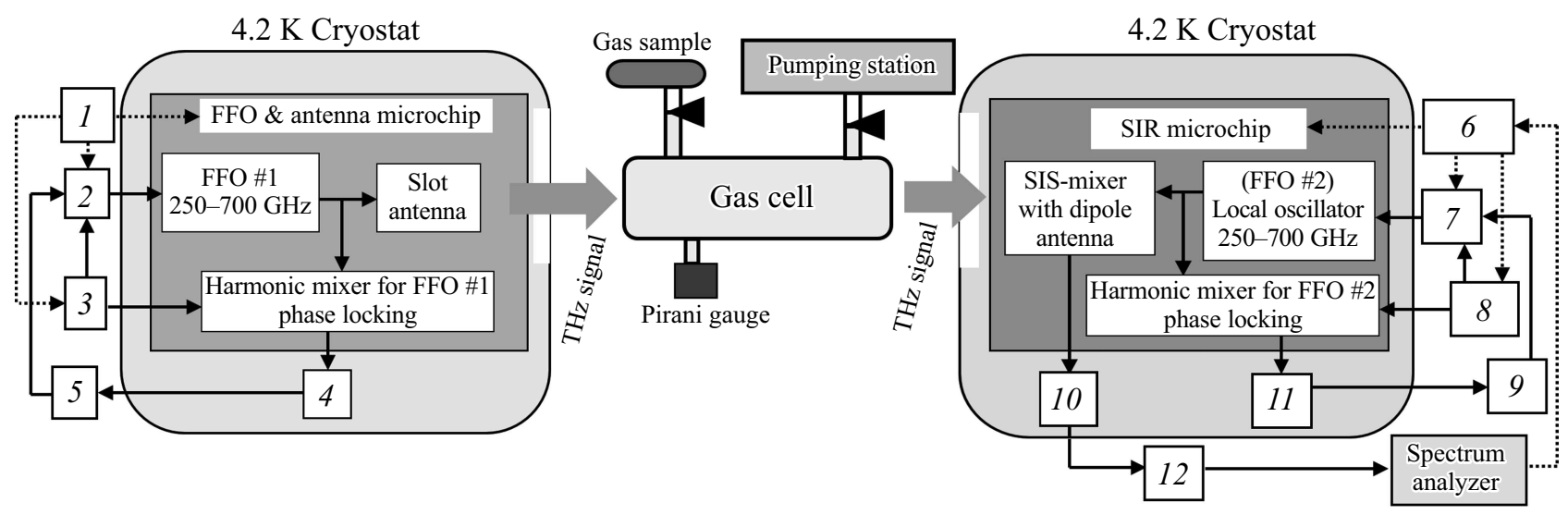

Рис. 1. Схема экспериментальной установки для регистрации линий поглощения газов в ТГц диапазоне на основе РДП-источника (FFO) и спектрометра на основе СИП (англ. SIR — от „superconducting integrated receiver“). Подписаны основные элементы схемы; вспомогательные элементы: 1,6 - персональный компьютер; 2, 7 - система ФАПЧ; 3, 8 - опорные генераторы СВЧ: $20 \mathrm{GHz}$ на гармонической смеситель и $400 \mathrm{MHz}$ на ФАПЧ; 4, 10,11 - криогенные $(4.2 \mathrm{~K})$ усилители сигнала ПЧ СИС-смесителей на базе транзисторов на высокой подвижности электронов; 5, 12 - усилители сигнала ПЧ гармонического смесителя диапазона 0-800 MHz при комнатной температуре; 9 - усилитель сигнала ПЧ диапазона 4-8 GHz при комнатной температуре.

до $700 \mathrm{GHz}$ с возможностью непрерывной перестройки путем управления локальным магнитным полем и током смещения. Исследования характеристик излучения источника проводились тремя принципиально разными методами: при помощи внешнего широкополосного охлаждаемого болометра на основе кремния [9-11], при помощи ТГц-спектрометра с разрешением порядка $40 \mathrm{kHz}$ на основе СИС-приемника с гетеродином на РДП [7,8], а также при помощи системы обратной связи с генератором посредством гармонического смесителя и системы ФАПЧ [7,8]. Каждый метод использовался для изучения различных характеристик.

Разработанный генератор представляется практичным решением в качестве внешнего источника ТГц-диапазона для применения в спектроскопии, микроскопии, построения изображений. В настоящей работе мы демонстрируем применимость такого источника для задач спектроскопии в эксперименте по регистрации линий поглощения газов. Наиболее близким применением показанной методики является исследование состава выдыхаемого воздуха, на основе которого возможно выявление веществ-маркеров и диагностика некоторых заболеваний [12-14].

\section{2. Система регистрации газов на основе ТГц-спектроскопии и метод исследования}

Система для регистрации линий поглощения газов основана на активном твердотельном источнике ТГц-излучения на базе РДП, подробно описанном, например, в работах $[7,10]$, и сверхпроводниковом интегральном приемнике (СИП) с высокой чувствительностью на базе СИС-перехода [15-16]. Схема комплексной мно- гоэлементной системы показана на рис. 1. Похожая схема была использована, например, в работе [17] с активным источником на основе лампы обратной волны, а также в работах [18-19] с активным источником на основе высокотемпературных монокристаллических сверхпроводников. Выходной „начальный“ сигнал РДП № 1 (англ. FFO \#1 - от „flux-flow oscillator“) проходит через измерительную ячейку с исследуемым газом и детектируется ТГц-спектрометром после некоторого поглощения, вызванного вращательными переходами молекул газа. Длина ячейки составляет $60 \mathrm{~cm}$, окна ячейки выполнены из тефлона, прозрачного в ТГц-диапазоне. Ширина линии поглощения в значительной степени зависит от давления газа, которое устанавливается скоростью напуска и откачки и измеряется вакуумметром Пирани. Следует отметить, что в системе имеется дополнительное поглощение сигнала воздухом в помещении, преимущественно водяным паром, которое, тем не менее, не влияло на эксперимент благодаря слишком широкой линии при атмосферном давлении, которая вносит однородный вклад в поглощение в частотной полосе анализа и не влияет на форму регистрируемого спектра. Полоса промежуточных частот (ПЧ) приемника составляет 4-8 GHz с центром, соответственно, при $6 \mathrm{GHz}$. Спектр поглощенного сигнала в конечном итоге регистрируется анализатором спектра в диапазоне ПЧ.

Методика регистрации спектров поглощения следующая. Частота гетеродина приемника (FFO \#2 на рис. 1) устанавливается на $6 \mathrm{GHz}$ выше либо ниже частоты поглощения исследуемого газа (например, $578.5 \mathrm{GHz}$ для частоты поглощения аммиака $572.5 \mathrm{GHz}$ ), а частота активного источника (FFO \#1 на рис. 1) плавно изменяется в узком диапазоне (например, $\pm 0.5-1 \mathrm{GHz}$ ) в окрестности частоты поглощения в обоих направлениях. Анализатор спектра работает в режиме записи 

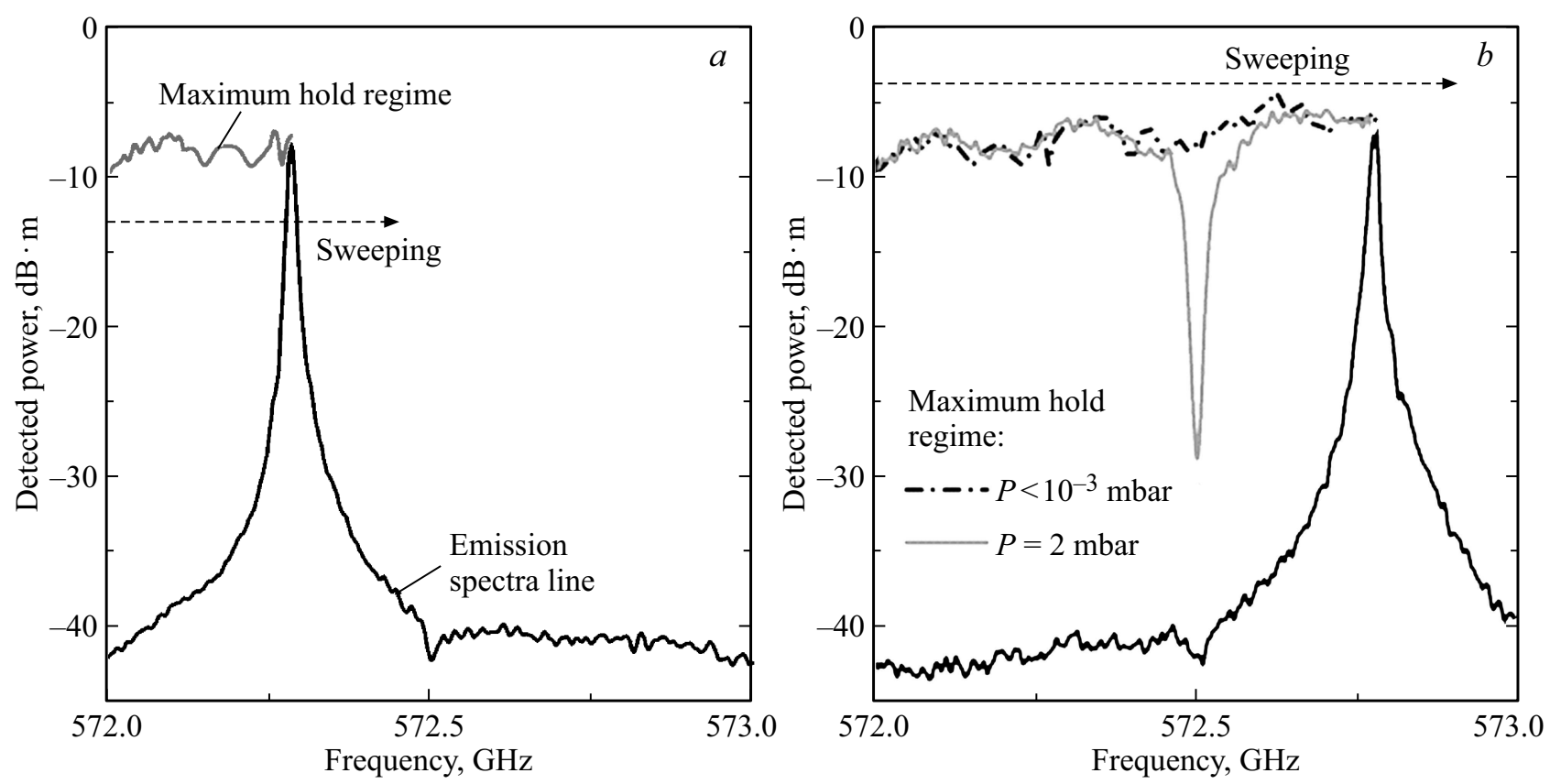

Рис. 2. Процесс регистрации линии поглощения газа в режиме записи максимального значения пика анализатором спектра методом „сканирования“ частоты слева направо: $a$ ) частота излучения генератора еще не достигла частоты поглощения; $b$ ) частота излучения генератора превысила частоту поглощения.

максимального значения пика линии излучения, так что на каждой частоте „сканирования“ регистрируется мощность пика, как показано на рис. 2, a. Если газ присутствует в измерительной ячейке и его давление достаточно для обнаружения, на регистрируемой кривой наблюдается четко выраженный минимум, как показано на рис. $2, b$. Когда ячейка откачана до высокого вакуума, анализатор спектра записывает относительно плоскую частотную зависимость (см. штрих-пунктирную линию на рис. $2, b)$, которая используется в дальнейшем как опорная кривая для обработки результатов. Поскольку выходной сигнал ПЧ не является идеально равномерным по частоте вследствие собственных частотных характеристик усилителей СВЧ и наличия стоячих волн в выходном тракте приемника, регистрируемая кривая максимального значения пика в отсутствие газа (опорная кривая) также не является идеально „плоской“. Данная процедура повторяется несколько раз при различном давлении газа, после чего из регистрируемой кривой при каждом давлении вычитается опорная кривая, чтобы привести сигнал в отсутствие поглощения к уровню $0 \mathrm{~dB}$.

\section{3. Результаты}

Результаты детектирования линий поглощения аммиака $\left(\mathrm{NH}_{3}\right)$ и воды $\left(\mathrm{H}_{2} \mathrm{O}\right)$ на частотах 572.5 и $556.9 \mathrm{GHz}$, соответственно, представлены на рис. 3. В качестве пробы аммиака использован доступный медицинский водный десятипроцентный раствор, следовательно, парциальное давление собственно аммиака ниже общего давления смеси, регистрируемого вакуумметром. В качестве пробы воды использована дистиллированная вода, поэтому регистрируемое давление является истинным давлением водяных паров с поправкой на погрешность измерения и калибровочные коэффициенты датчика Пирани для разных газов. Напуск газа в ячейку осуществляется путем естественного испарения жидкости в пробирке, соединенной с входным патрубком ячейки, через игольчатый натекатель с плавной регулировкой. Перед напуском газа ячейка предварительно откачивалась до давления ниже $10^{-5} \mathrm{mbar}$ (предел измерения вакуумметра Пирани) в течение $10-15 \mathrm{~min}$. Рис. 3 наглядно демонстрирует уширение спектров и увеличение мощности поглощения с ростом давления газа. Характерная полуширина линии поглощения аммиака при давлении $0.5 \mathrm{mbar}$ составила около $8 \mathrm{MHz}$. Характерное давление в эксперименте варьировалось от $\sim 10^{-3}$ до порядка 20 mbar: при более высоком давление линия поглощения по-прежнему была наблюдаема, но ее спектральная ширина была слишком большой вследствие столкновительного уширения. Предельные давления для обнаружения газов в проведенном эксперименте составили около $0.05 \mathrm{mbar}$ для аммиака и $0.005 \mathrm{mbar}$ для воды. Длительность одного измерения, равное времени „Прохода“ частоты вверх и обратно вниз, составляла около 2 минут для каждого значения давления.

Интересно отметить тот факт, что ширина линии активного источника на основе РДП практически неважна в данном эксперименте, важно лишь, чтобы шаг „сканирования“ по частоте был меньше ширины линии для более точной регистрации кривых в режиме записи 

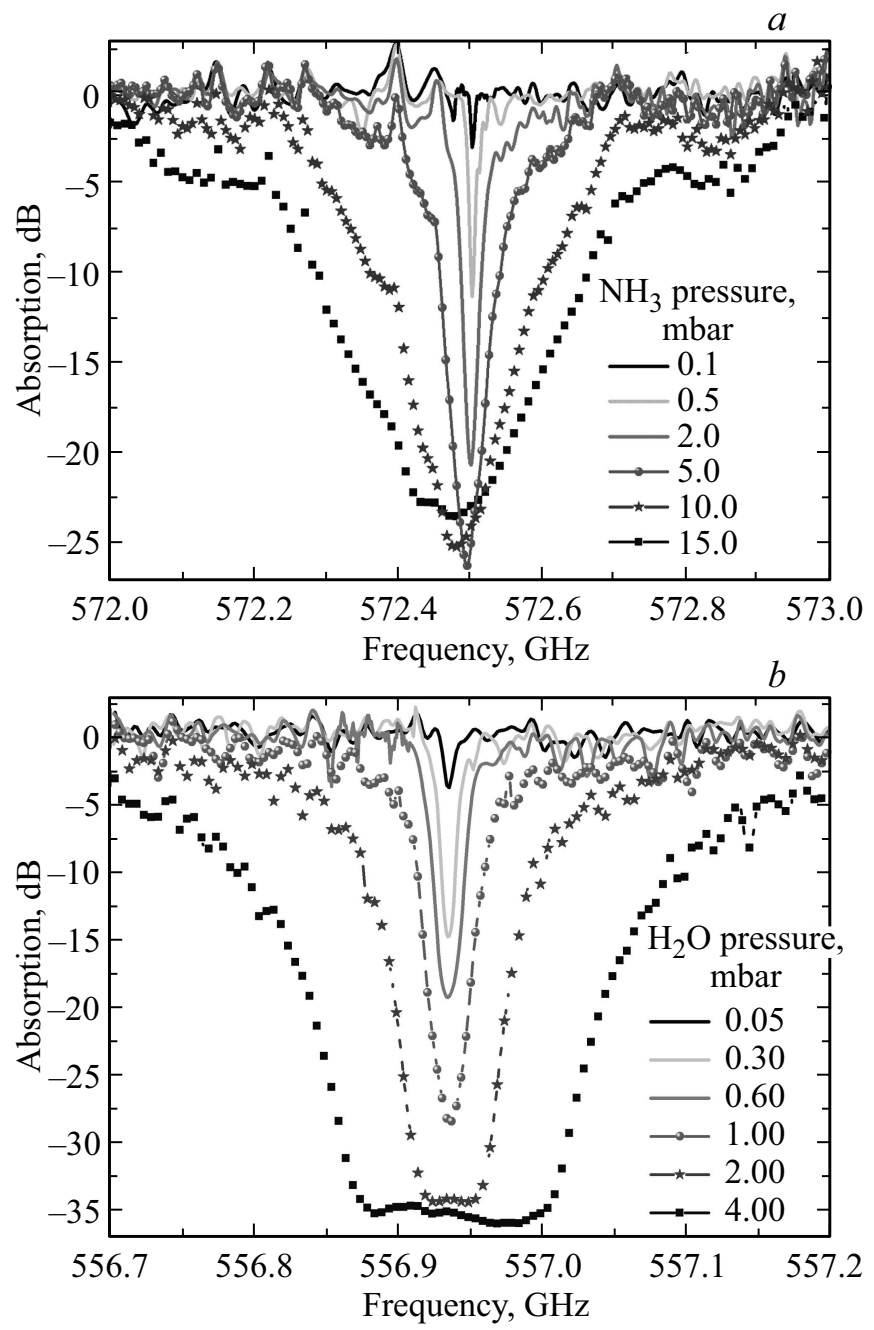

Рис. 3. Спектры поглощения, зарегистрированные системой на основе РДП-источника и ТГц-спектрометра при различном уровне давления $a$ ) аммиака, водный раствор $10 \%$; $b$ ) дистиллированной воды.

максимального значения пика, а также чтобы время нахождения генератора в каждой точке по частоте было достаточным с учетом скорости записи анализатора спектра. Так, характерная ширина линии в эксперименте с аммиаком составляла около $15 \mathrm{MHz}$ (спектр линии показан на рис. 2) и около $12 \mathrm{MHz}$ в эксперименте с водой. Ширина линии источника начинает играть роль лишь в том случае, когда излучение приводит к насыщению приемника, что может влиять на точность результатов. Стабилизация источника на основе системы ФАПЧ и гармонического смесителя предусмотрена, но не используется в данной методике „сканирования“ по частоте. Полученные характеристики - частота поглощения, мощность, ширина спектральных линий - хорошо соответствуют справочной литературе и табличным значениям.

Спектральное разрешение предложенного метода определяется разрешением СИП со стабилизованным по фазе гетеродином на основе собственного РДП; это разрешение лучше, чем $100 \mathrm{kHz}$ [15]. Проведенный эксперимент демонстрирует спектральное разрешение и чувствительность данной системы регистрации газов, близкие к характеристикам других систем либо превосходящие их - например, систем на основе квантовокаскадных лазеров из работы [20], Фурье-спектрометра на чирпированных импульсах [21]. Наконец, стоит отметить, что чувствительность по давлению газов, продемонстрированная в настоящей работе, может быть улучшена на один-два порядка при использовании другой системы считывания и при дополнительной модуляции активного источника на основе РДП. Так, в настоящей работе использован анализатор спектра в качестве оконечного устройства для регистрации кривых поглощения, при этом наличие либо отсутствие газа оценивалось визуально по экрану анализатора оператором установки. С точки зрения чувствительности, данный метод далек от предельно достижимых параметров. Для повышения чувствительности можно использовать измеритель СВЧ-мощности либо спектрометр на основе быстрого преобразования Фурье, а также накапливать сигнал долгое время и увеличивать число „проходов“ линии поглощения при сканировании частоты. Тем не менее, проведенный эксперимент демонстрирует потенциальную применимость системы ТГц-спектроскопии газов для целого круга задач, таких как медицинская диагностика при помощи регистрации веществ-маркеров в выдыхаемом воздухе, контроль технологических процессов и утечек газов, экологической мониторинг.

\section{4. Заключение}

В настоящей работе мы разработали систему детектирования газов в газовой смеси на основе спектроскопии поглощения в ТГц-диапазоне частот. В качестве активного источника был использован разработанный нами ранее широко перестраиваемый твердотельный генератор на основе РДП. В качестве детектора был использован сверхпроводниковый интегральный приемник на основе СИС-перехода, а конечным устройством анализа являлся анализатор спектра, работающий в полосе выходных частот приемника 4-8 GHz. На основе данной системы продемонстрирована регистрация линии поглощения аммиака на частоте $572.5 \mathrm{GHz}$ с предельно регистрируемым давлением порядка $0.05 \mathrm{mbar}$, и воды на частоте $556.9 \mathrm{GHz}$ с предельно регистрируемым давлением порядка 0.005 mbar. Предложены области применения данной методики газового анализа, такие как диагностика некоторых заболеваний по исследованию выдыхаемого воздуха, технологический и экологический мониторинг.

\section{Финансирование работы}

Исследование выполнено за счет гранта Российского научного фонда (проект № 17-79-20343-П). Изготовле- 
ние образцов РДП и СИП выполнено с использованием уникальной научной установки (УНУ № 352529) ИРЭ им. В.А. Котельникова РАН, созданной и функционирующей за счет бюджетного финансирования в рамках государственного задания.

\section{Конфликт интересов}

Авторы заявляют, что у них нет конфликта интересов.

\section{Список литературы}

[1] S.L. Dexheimer. Terahertz Spectroscopy: Principles and Applications. CRC Press, N. Y. (2008). 360 p.

[2] M.C. Beard, G.M. Turner, C.A. Schmuttenmaer. Terahertz Spectroscopy. J. Phys. Chem. B 106, 29, 7146 (2002).

[3] J.B. Baxter, G.W. Guglietta. Terahertz Spectroscopy. Anal. Chem. 83, 12, 4342 (2011).

[4] A.G. Davies, A.D. Burnett, W. Fan, E.H. Linfield, J.E. Cunningham. Terahertz spectroscopy of explosives and drugs. Mater. Today 11, 3, 18 (2008).

[5] D.F. Plusquellic, K. Siegrist, E.J. Heilweil, O. Esenturk. Applications of Terahertz Spectroscopy in Biosystems. Chem. Phys. Chem. 8, 17, 2412 (2007).

[6] Н.В. Кинев, К.И. Рудаков, А.М. Барышев, В.П. Кошелец. ФTT 60, 11, 2132 (2018).

[7] N.V. Kinev, K.I. Rudakov, L.V. Filippenko, A.M. Baryshev, V.P. Koshelets. J. Appl. Phys. 125, 15, 151603 (2019).

[8] Н.В. Кинев, К.И. Рудаков, Л.В. Филиппенко, А.М. Барышев, В.П. Кошелец. Радиотехника и электроника 64, 10, 970 (2019).

[9] N.V. Kinev, K.I. Rudakov, L.V. Filippenko, A.M. Baryshev, V.P. Koshelets. IEEE Trans. THz Sci. Technol. 9, 6, 557 (2019).

[10] Н.В. Кинев, К.И. Рудаков, Л.В. Филиппенко, В.П. Кошелец, A.M. Барышев. ФТТ 62, 9, 1379 (2020).

[11] N.V. Kinev, K.I. Rudakov, L.V. Filippenko, A.M. Baryshev, V.P. Koshelets. Sensors 20, 24, 7267 (2020).

[12] T. Higenbottam. Exp. Physiol. 80, 5, 855 (1995).

[13] K. Alving, E. Weitzberg, J.M. Lundberg. Eur. Res. J. 6, 9, 1368 (1993).

[14] Е.В. Степанов. Диодная лазерная спектроскопия и анализ молекул-биомаркеров. Физматлит, М. (2009). 416 с.

[15] G.de Lange, M. Birk, D. Boersma, J. Dercksen, P. Dmitriev, A.B. Ermakov, L.V. Filippenko, H. Golstein, R.W.M. Hoogeveen, L. de Jong, A.V. Khudchenko, N.V. Kinev, O.S. Kiselev, B. van Kuik, A. de Lange, J. van Rantwijk, A.M. Selig, A.S. Sobolev, M.Yu. Torgashin, E. de Vries, G. Wagner, P.A. Yagoubov, V.P. Koshelets. Supercond. Sci. Technol. 23, 4, 045016 (2010).

[16] V.P. Koshelets, P.N. Dmitriev, M.I. Faley, L.V. Filippenko, K.V. Kalashnikov, N.V. Kinev, O.S. Kiselev, A.A. Artanov, K.I. Rudakov, A. de Lange, G. de Lange, V.L. Vaks, M.Y. Li, H. Wang. IEEE Trans. THz Sci. Technol. 5, 4, 687 (2015).

[17] N.V. Kinev, L.V. Filippenko, K.V. Kalashnikov, O.S. Kiselev, V.L. Vaks, E.G. Domracheva, V.P. Koshelets. J. Phys. Conf. Ser. 741, 12169 (2016).
[18] E. Sobakinskaya, V.L. Vaks, N. Kinev, M. Ji, M.Y. Li, H.B. Wang, V.P. Koshelets. J. Phys. D 50, 3, 035305 (2017).

[19] H. Sun, Z. Yang, N.V. Kinev, O.S. Kiselev, Y. Lv, Y. Huang, L. Hao, X. Zhou, M. Ji, X. Tu, C. Zhang, J. Li, F. Rudau, R. Wieland, J.S. Hampp, O. Kizilaslan, D. Koelle, B. Jin, J. Chen, L. Kang, W. Xu, R. Kleiner, V.P. Koshelets, H. Wang, P. Wu. Phys. Rev. Appl. 8, 5, 054005 (2017).

[20] L. Consolino, S. Bartalini, H.E. Beere, D.A. Ritchie, M.S. Vitiello, P. De Natale. Sensors 13, 3, 3331 (2013).

[21] E. Gerecht, K.O. Douglass, D.F. Plusquellic. Opt. Express 19, 9, 8973 (2011).

Редактор Е.Ю. Флегонтова 